\title{
Article \\ Nanoscale Superconducting States in the Fe-Based Filamentary Superconductor of Pr-Doped $\mathrm{CaFe}_{2} \mathrm{As}_{2}$
}

\author{
Giang D. Nguyen ${ }^{1,+}{ }^{+}$Mingming Fu ${ }^{1,2,+} \mathbb{D}$, Qiang Zou ${ }^{1}$, Liurukara D. Sanjeewa ${ }^{3}$, An-Ping Li ${ }^{1}$, Athena S. Sefat ${ }^{3}$ \\ and Zheng Gai ${ }^{1, *(D)}$
}

check for updates

Citation: Nguyen, G.D.; Fu, M.; Zou, Q.; Sanjeewa, L.D.; Li, A.-P.; Sefat,

A.S.; Gai, Z. Nanoscale

Superconducting States in the

Fe-Based Filamentary

Superconductor of Pr-Doped $\mathrm{CaFe}_{2} \mathrm{As}_{2}$. Nanomaterials 2021, 11 , 1019. https://doi.org/10.3390/ nano11041019

Academic Editor: Evgueni

F. Talantsev

Received: 24 March 2021

Accepted: 14 April 2021

Published: 16 April 2021

Publisher's Note: MDPI stays neutral with regard to jurisdictional claims in published maps and institutional affiliations.

Copyright: (c) 2021 by the authors. Licensee MDPI, Basel, Switzerland. This article is an open access article distributed under the terms and conditions of the Creative Commons Attribution (CC BY) license (https:/ / creativecommons.org/licenses/by/ $4.0 /)$.
1 Center for Nanophase Materials Sciences, Oak Ridge National Laboratory, Oak Ridge, TN 37831, USA; giangnguyen7386@gmail.com (G.D.N.); mmfu@ncu.edu.cn (M.F.); qzou.iphy@gmail.com (Q.Z.); apli@ornl.gov (A.-P.L.)

2 Department of Physics, Nanchang University, Nanchang 330031, China

3 Materials Science \& Technology Division Oak Ridge National Laboratory, Oak Ridge, TN 37831, USA; lsanjeew@utk.edu (L.D.S.); athena.sefat@science.doe.gov (A.S.S.)

* Correspondence: gaiz@ornl.gov

+ These authors contributed equally to this work.

Abstract: The low-temperature scanning tunneling microscope and spectroscopy (STM/STS) are used to visualize superconducting states in the cleaved single crystal of $9 \%$ praseodymium-doped $\mathrm{CaFe}_{2} \mathrm{As}_{2}$ (Pr-Ca122) with $T_{\mathcal{C}} \approx 30 \mathrm{~K}$. The spectroscopy shows strong spatial variations in the density of states (DOS), and the superconducting map constructed from spectroscopy discloses a localized superconducting phase, as small as a single unit cell. The comparison of the spectra taken at $4.2 \mathrm{~K}$ and $22 \mathrm{~K}$ (below vs. close to the bulk superconducting transition temperature) from the exact same area confirms the superconducting behavior. Nanoscale superconducting states have been found near Pr dopants, which can be identified using $d I / d V$ conductance maps at $+300 \mathrm{mV}$. There is no correlation of the local superconductivity to the surface reconstruction domain and surface defects, which reflects its intrinsic bulk behavior. We, therefore, suggest that the local strain of Pr dopants is competing with defects induced local magnetic moments; this competition is responsible for the local superconducting states observed in this Fe-based filamentary superconductor.

Keywords: iron-based superconductor; filamentary superconductor; nanoscale superconducting states; defects; scanning probe microscopy; defects; domain boundary

\section{Introduction}

Discovery of the high- $T_{c}$ of Fe-based superconductors has attracted much attention recently. Among the materials, 122-type iron-based superconductivity is of particular interest due to its relatively simple structure and the easy growth of large single crystals [1]. The parent compound of the 122 family exhibits antiferromagnetic order at low temperature, and the superconductivity typically emerges through chemically doping. However, the mechanism of doping-induced superconductivity in 122 superconductors is still controversial. Additionally, the superconducting doped samples are electronically inhomogeneous, even on a nanoscale. Therefore, a scanning probe microscope has been an ideal tool to study the doping effects as well as the superconducting mechanism on these Fe-based superconductors [2]. The majority of recent research in this area focuses on bulk superconducting samples, and only limited studies have been conducted on filamentary superconducting behavior. It has been argued that the filamentary superconductors arise due to spontaneous electronic inhomogeneity at the nanoscale level of the sample, and STM/S might be the most adequate tool to reveal the insight to filamentary behavior [3,4]. Such electronic inhomogeneities may be caused by nanoscale dopants and disorder, which manifest in non-zero resistance and have a very small Meissner effect (field-cooled in magnetic susceptibility), although their shielding is the same as bulk superconductors $[5,6]$. 
As an example of the neglected filamentary superconductor, electron-doped, Pr-doped $\mathrm{CaFe}_{2} \mathrm{As}_{2}$ (Pr-Ca122) crystal shows the highest transition temperature among the pnictides with $\mathrm{ThCr}_{2} \mathrm{Si}_{2}$ crystal structure $\left(T_{c} \sim 45 \mathrm{~K}\right)$. The difficulty in using STM to achieve this goal is how to identify the dopant position and correlate it to superconducting states. Recently, Gogryky et al. studied the optimal-doped Pr-Ca122 crystal (14\% dopant) and found the local electronic inhomogeneous; [3] Zeljkovic et al., demonstrated a method for identifying the $\mathrm{Pr}$-dopant location on a filamentary superconductor of $\mathrm{Pr}$-doped $\mathrm{CaFe}_{2} \mathrm{As}_{2}$ (Pr-Ca122) [4]. However, there was no STM spectroscopy performed to correlate this dopant location with superconducting states.

Here, we use scanning tunneling microscopy and spectroscopy (STM/STS) to study local superconducting states on lower Pr-doped Ca122 crystal (9\% dopant) to avoid segregated dopant clusters and to corelate filamentary superconductivity to local structure and dopants on a nanoscale.

\section{Materials and Methods}

Single crystals of Pr-doped $\mathrm{CaFe}_{2} \mathrm{As}_{2}$ (Pr-Ca122 were grown out of FeAs self-flux technique, similar to ref. [1,7], with [001] direction perpendicular to the crystalline plate shapes. The chemical composition of the crystals was measured with a Hitachi S3400 scanning electron microscope energy-dispersive $X$-ray spectroscopy (EDS). The structures were identified as tetragonal ThCr2Si2 type (I4/ $\mathrm{mmm}, \mathrm{Z}=2)$ at room temperature, and lattice parameters upon doping were refined using $X^{\prime}$ Pert HighScore by collecting data on an X'Pert PRO MPD X-ray powder diffractometer. Magnetic data were collected using Quantum design's magnetic property measurement system (MPMS).

Single crystals of Pr-Ca122 were cleaved at liquid nitrogen temperature in an ultrahigh vacuum system, then immediately transferred into an in-situ STM precooled to $4.2 \mathrm{~K}$ without breaking vacuum. The STM/S experiments were carried out using a scanning tunneling microscope with base pressure better than $2 \times 10^{-10}$ Torr, with a chemically etched W tip. All W tips were conditioned on clean Au (1 111$)$ and checked using the topography, surface state, and work function before each measurement. The STM/S were controlled by the SPECS Nanonis control system. Topographic images were acquired in constant current mode with bias voltage applied to samples and tip grounded. All the spectroscopies were obtained using the lock-in technique with a modulation of $1 \mathrm{mV}$ at $973 \mathrm{~Hz}$ on bias voltage, $d I / d V$. Current-imaging-tunneling-spectroscopy was collected over a grid of pixels at bias ranges around Fermi level using the same lock-in amplifier parameters.

\section{Results and Discussion}

The crystal structure of the parent compound of Pr-doped $\mathrm{CaFe}_{2} \mathrm{As}_{2}$ (Pr-Ca122) is shown in Figure 1a. It composed of a trilayer building block of FeAs sandwiched between checkerboard layers of $\mathrm{Ca}$, with lattice constants of $\mathrm{a}=\mathrm{b}=0.395 \mathrm{~nm}$ and $\mathrm{c}=1.3 \mathrm{~nm}$. A small amount (9\% measured concentration in this work) of $\mathrm{Pr}$ is used to dope the compound by substituting at the $\mathrm{Ca}$ site. The temperature dependence of magnetic susceptibility in the zero-field cool measurement of the Pr-Ca122 sample is shown in Figure 1b. The downturn of the susceptibility signifying diamagnetic responses as a result of the Meissner state reveals a superconducting transition at $30 \mathrm{~K}$. However, the sample is only considered a filamentary superconductor due to its small superconducting volume fraction $(\sim 3 \%$ at 5 K) [3,8-10]. In order to visualize the local superconductivity behavior in Pr-Ca122, we performed a STM study on in situ cleaved samples. Figure 1c shows a typical large-scale STM morphology image of the cleaved surface. The step height of $\sim 0.65 \mathrm{~nm}$ (line profile in the inset) is about a half of the unit cell size along the $\mathrm{c}$ axis. It reflects the mirror symmetry of Ca122 crystal structure (Figure 1a). 
(a)

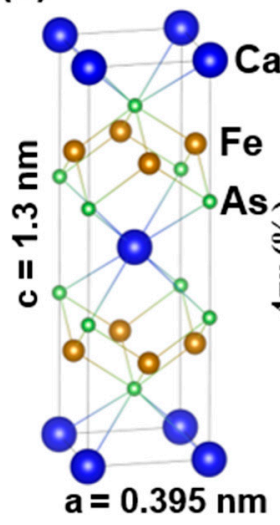

(b)

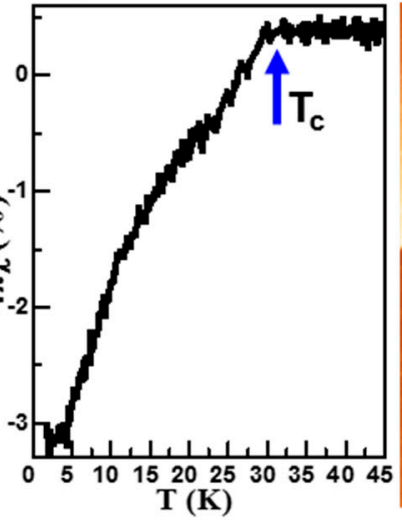

(c)

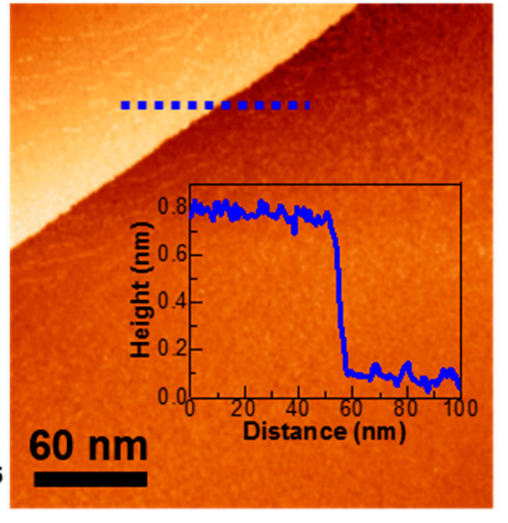

Figure 1. (a) Crystal structure of Ca122. (b) Temperature dependence of zero-field-cooled magnetic susceptibility of the sample of Pr-Ca122, showing diamagnetism below $30 \mathrm{~K}$, under a constant magnetic field of 20 Oe. (c) Large scale STM topographic image after cleaving (sample bias $V_{S}=-20 \mathrm{mV}$, tunneling current $I t=100 \mathrm{pA}$, temperature $T=4.2 \mathrm{~K}$ ). The line profile across a half unit cell step is shown at the inset.

An atomic resolution STM image (Figure 2a) demonstrates a stripy pattern with a periodicity of $0.80 \pm 0.02 \mathrm{~nm}$, which is the double unit cell in the ab plane. It suggests the surface is terminated by $\mathrm{Ca}$ atoms with a $2 \times 1$ reconstruction [4]. To identify the $\mathrm{Pr}$ dopants on the surface terminated by Ca atoms, we applied a similar method discussed in the previous report [4]. The Pr dopants appear as a bright area in a $d I / d V$ conductance at a high positive sample bias of $300 \mathrm{mV}$ (marked by dashed white rings in Figure 2b). The $d I / d V$ maps at the negative sample biases (Figure $2 \mathrm{c}, \mathrm{d}$ ) do not show any distinctive contrast.
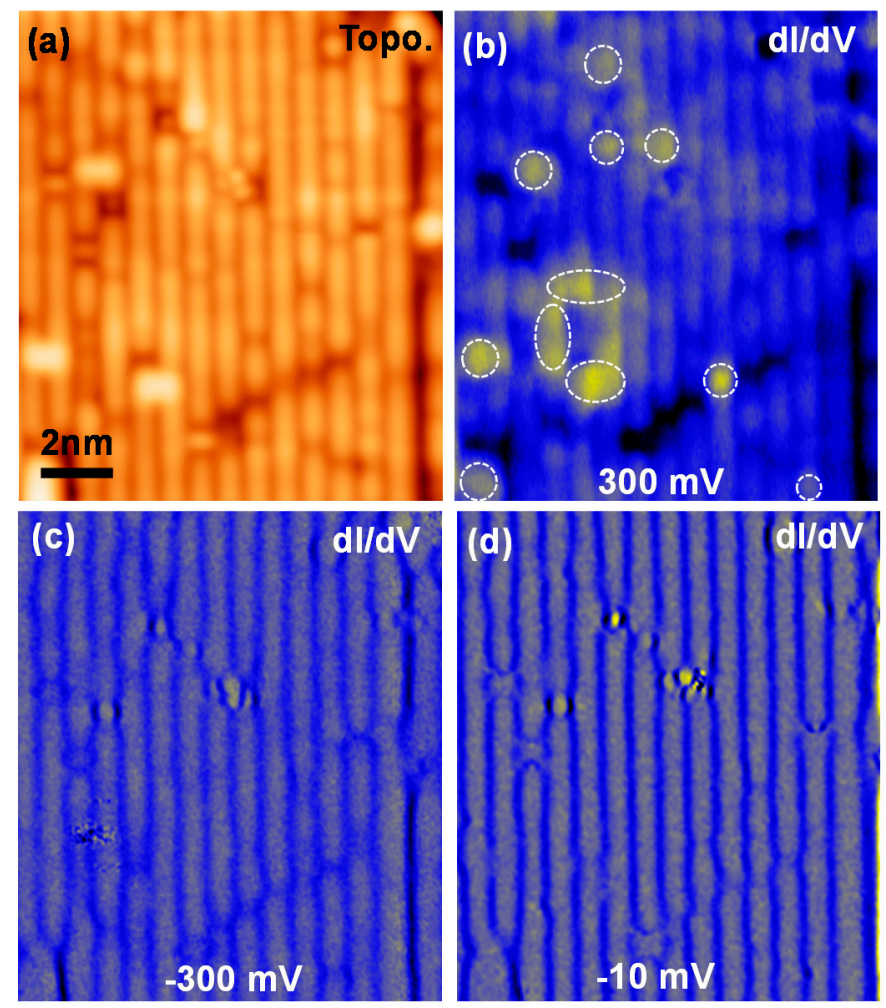

Figure 2. (a,b) STM image and $d I / d V$ conductance map on Pr-Ca122 (Vs $=300 \mathrm{mV}$, It $=200 \mathrm{pA}$, modulation voltage $V a c=1 \mathrm{mV}, f=973 \mathrm{~Hz}, T=4.2 \mathrm{~K}$ ). The bright spots on the $d I / d V$ map in (b) indicate the Pr-dopant positions (dashed white circles). (c,d) Same area with $V s=-300 \mathrm{mV}$ and $-10 \mathrm{mV}$, respectively. 
As the global susceptibility result of Pr-Ca122 shows filamentary superconducting behavior, it is interesting to reveal how filamentary superconductivity corelates with local structures. When surveying around the samples, we find that although STS $d I / d V$ curves show gap-like features in occasional spots, STS from majority areas do not show superconducting gap. To visualize the local superconductivity, a superconducting gap map serves better than an STS $d I / d V$ map. As an example, Figure 3 shows a STM topographic image and corresponding superconducting gap map, which is calculated from the current imaging tunneling spectroscopy (CITS) image at $4.2 \mathrm{~K}[11,12]$. The majority area of the sample does not show superconductivity, which is consistent with a low superconducting volume fraction from the bulk measurement (about 3\%). The localized superconducting areas are observed mostly near Pr dopants, which we can identify as Figure 2b (Figure 3b, white rings). Surprisingly, the superconducting state is very localized, which can be in the range of a few cell-size units of the compound $(a=0.395 \mathrm{~nm})$. The typical extracted $d I / d V$ spectra across a superconducting region (blue arrow in Figure 3a) are presented in Figure 3c. The spatial distance between each curve is $0.12 \mathrm{~nm}$. The five red curves show superconducting states, which have a superconducting symmetric gap at Fermi level with coherent peaks. Away from the superconducting region, the $d I / d V$ spectra show normal metallic states (black curves). In between the red and black curves, there are a couple of curves that show pseudogap states (green curves); they are the mixing between superconducting and normal states [12]. The local superconducting area at this position is around $1 \mathrm{~nm}$.
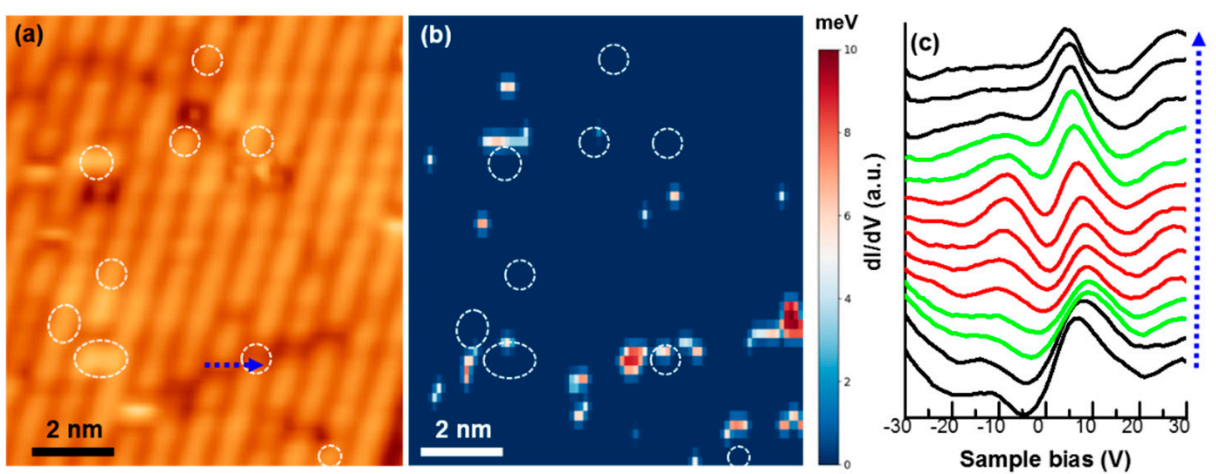

Figure 3. (a,b) STM image and superconducting gap map respectively extracted from CITS measurement at $4.2 \mathrm{~K}$ on the surface area, as shown Figure $2 \mathrm{a}\left(V_{S}=-10 \mathrm{mV}, I t=200 \mathrm{pA}, V_{a c}=0.5 \mathrm{mV}\right.$, $f=973 \mathrm{~Hz}, T=4.2 \mathrm{~K}$ ). The white dashed rings mark the position of Pr dopants, as found on the $d I / d V$ map in Figure $2 \mathrm{~b}$. The distortion of the image compared with Figure 2 is due to the thermal drift of $15 \mathrm{~h} \mathrm{CITS.} \mathrm{(c)} d I / d V$ line spectra taken across a superconducting phase area marked by the blue arrow in (a). The spatial distance between each taken curve is $0.12 \mathrm{~nm}$. Red and black mark the superconducting density of states and normal metallic density of states, respectively. The transition curves from the superconducting density of states to the normal metallic density of states have pseudogap characteristics, which are marked with a green color. Vertical offsets are applied to the spectra for clarity.

There are some areas on the surface with higher Pr dopant concentrations, mostly around defective regions and domain boundaries. Figure 4a,b show the STM topographic image and $d I / d V$ map, respectively, in such a region, with Pr dopants (yellow spots and lines) along bulk domain boundaries and nearby strained areas. Figure $4 \mathrm{c}$ displays the $d \mathrm{I} / d V$ line spectra at a temperature of $6 \mathrm{~K}$ along a black arrow in Figure 4a. The curves show a gap-like feature, which presents the superconducting and pseudogap phase. At an increased temperature of $22 \mathrm{~K}$, the $d I / d V$ line spectra recorded in the sample region show strongly suppressed superconducting gap states (Figure 4c). This is consistent with the bulk measurement of the superconducting transition temperature of the sample at about $30 \mathrm{~K}$ (Figure 1b). 

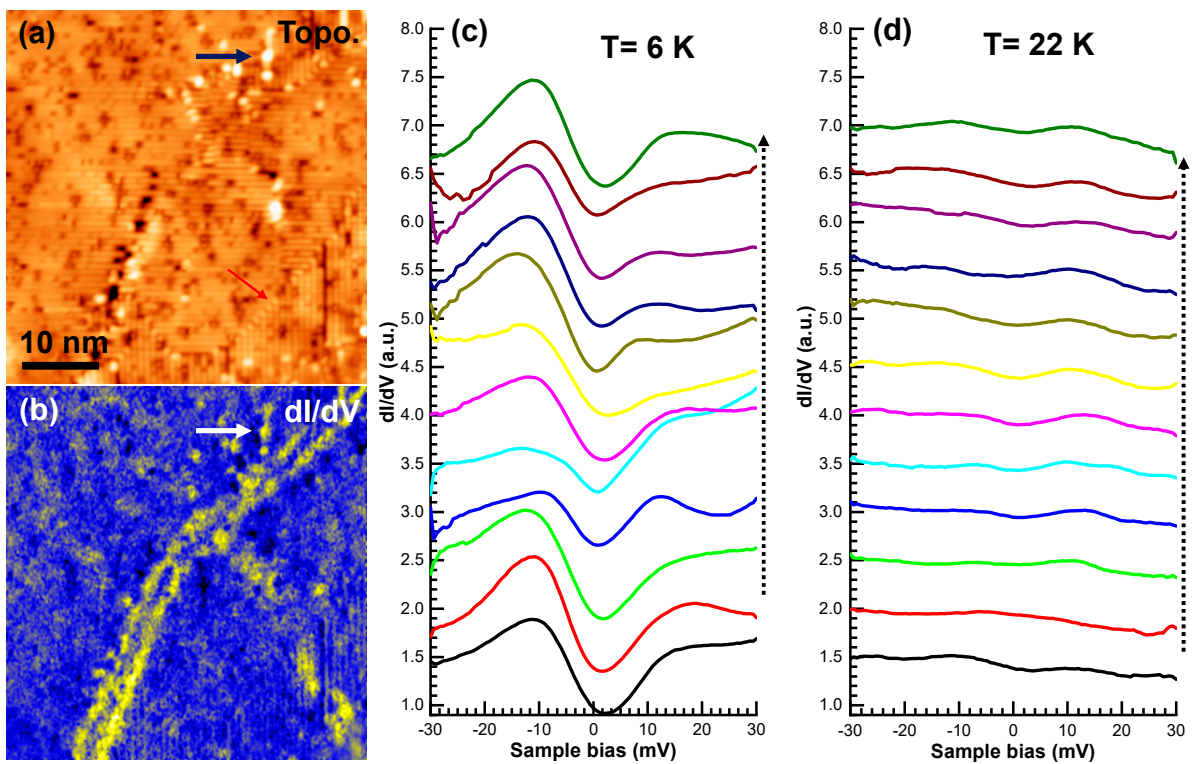

Figure 4. (a,b) STM image and $d I / d V$ conductance map on a Pr-Ca122 region $(V s=300 \mathrm{mV}$, It $=200 \mathrm{pA}$, $\left.V_{a c}=1 \mathrm{mV}, f=973 \mathrm{~Hz}, T=6 \mathrm{~K}\right) .(\mathbf{c}, \mathbf{d}) d \mathrm{I} / d V$ line spectra along the dotted arrow on the same area at $6 \mathrm{~K}$ and $22 \mathrm{~K}$, respectively $\left(V_{s}=-10 \mathrm{mV}\right.$, It $\left.=200 \mathrm{pA}, V_{a c}=0.5 \mathrm{mV}, f=973 \mathrm{~Hz}\right)$. The same vertical offset is applied to the spectra in both $(\mathbf{c}, \mathbf{d})$. The red arrow points to a reconstruction of the domain boundary.

It is important to note that the domain boundaries and defective regions where $\mathrm{Pr}$ dopants segregate are intrinsic bulk-like structures, which are created during crystal growth. Those sites are different from the $2 \times 1$ surface reconstruction 90-degree domain boundaries, as pointed out by a red arrow in Figure 4a. As shown in Figure 4b, Pr dopant concentration near reconstruction domain boundaries is similar to regular areas, as these boundaries are the result of restructuring surface $\mathrm{Ca} / \mathrm{As} / \mathrm{Pr}$ atoms during exposure of the surface at cleavage.

We also notice the local superconductivity observed in this work is not originated from surface-related defects. Shown in Figure 5a are some examples of local defects. The STS map of the area shows no Pr dopants around. The STS $d I / d V$ spectra for defects 1,2 , 3 and the non-defected area are shown in Figure 5b. Although defects areas are different from the pristine area, none of them show superconducting gap or pseudogap features.
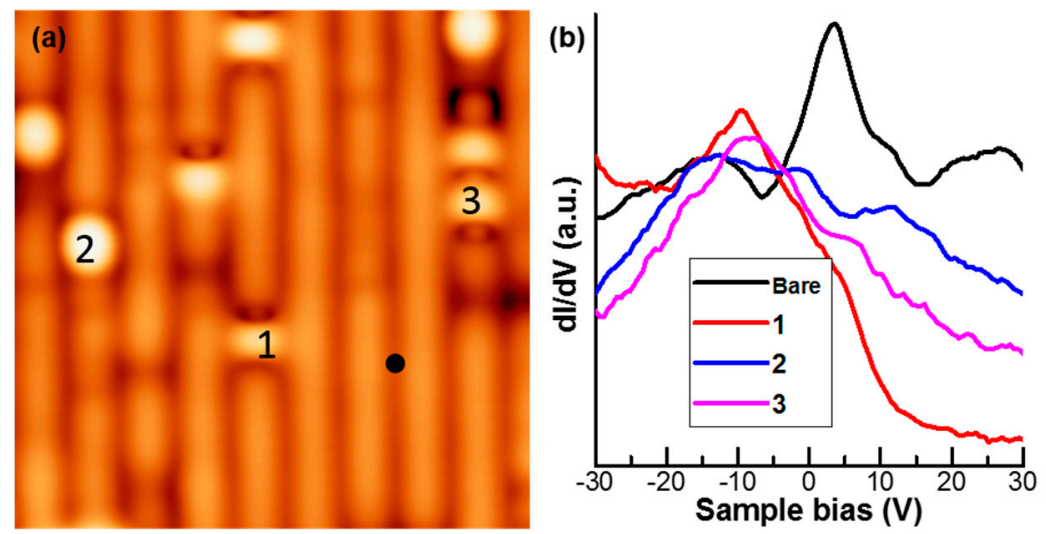

Figure 5. (a) STM atomic resolution image in a pristine area of Pr-Ca122 surface with $\left(V_{S}=-2 \mathrm{mV}\right.$, It $=200 \mathrm{pA}, T=4.2 \mathrm{~K})$. (b) The $d I / d V$ spectra taken at three different pristine defects are very different from one taken at the bare area, and they all show no superconducting gap.

Here we discuss the role of $\operatorname{Pr}$ dopants in response to local superconductivity in Pr-Ca122. This observation of local superconducting states near Pr dopants supports the 
hypothesis of the superconducting phase induced by local strains near Pr dopants [13-16]. The local strain can yield the optimum Fe-As bonding angle for emergence of the superconducting phase [17]. However, these Pr dopants, in parallel, create lattice defects with some net magnetic moment [18]. The local magnetic moment can also destroy superconductivity [11]. Therefore, the Pr-Ca122 sample is not able to become a bulk superconductor by simply increasing the Pr dopant concentration. These two competing processes result in the filamentary superconductivity observed in Pr-Ca122.

\section{Conclusions}

Low-temperature STM/STS is used to observe nanoscale superconducting states in the cleaved single crystal of low $\mathrm{Pr}$-doped $\mathrm{CaFe}_{2} \mathrm{As}_{2}$. The spectroscopy shows strong spatial variations of density of states, and a superconducting map constructed from spectroscopy discloses a localized superconducting phase, as small as a single unit cell. The comparison of the spectra taken at low temperature $(4.2 \mathrm{~K})$ and an elevated temperature $22 \mathrm{~K}$, which is below but near the bulk transition temperature area, confirms superconducting behavior. Nanoscale superconducting states have been found near Pr dopants, which can be identified using $d I / d V$ conductance maps at $+300 \mathrm{mV}$. Local superconductivity is not influenced by surface reconstruction and surface defects created during cleavage. We, therefore, suggest that the competition between the local strain at Pr dopants and the local magnetic moment induced by defects is responsible for the local superconducting states observed in this Fe-based filamentary superconductor.

Author Contributions: Conceptualization, A.S.S. and Z.G.; methodology, STM/STS, G.D.N., M.F., Q.Z., A.-P.L. and Z.G., crystal growth, L.D.S. and A.S.S.; writing, G.D.N. and Z.G. All authors have read and agreed to the published version of the manuscript.

Funding: This research received no external funding.

Institutional Review Board Statement: Not applicable.

Informed Consent Statement: Not applicable.

Data Availability Statement: The data presented in this study are available on request from the corresponding author.

Acknowledgments: This research was conducted at the Center for Nanophase Materials Sciences, which is a DOE Office of Science User Facility. G.D.N., L.D.S., A.S.S. and part of Z.G.'s research in this work was supported by the U.S. Department of Energy (DOE), Office of Science, Basic Energy Sciences (BES), Materials Science and Engineering (MSE) Division.

Conflicts of Interest: The authors declare no conflict of interest.

Disclaimer: This manuscript has been authored by UT-Battelle, LLC under Contract No. DEAC05-00OR22725 with the U.S. Department of Energy. The United States Government retains and the publisher, by accepting the article for publication, acknowledges that the United States Government retains a non-exclusive, paid-up, irrevocable, world-wide license to publish or reproduce the published form of this manuscript, or allow others to do so, for United States Government purposes. The Department of Energy will provide public access to these results of federally sponsored research in accordance with the DOE Public Access Plan.

\section{References}

1. Mandrus, D.; Sefat, A.S.; McGuire, M.A.; Sales, B.C. Materials chemistry of $\mathrm{BaFe}_{2} \mathrm{As}_{2}$ : A model platform for unconventional superconductivity. Chem. Mater. 2010, 22, 715-723. [CrossRef]

2. Hoffman, J.E. Spectroscopic scanning tunneling microscopy insights into Fe-based superconductors. Rep. Prog. Phys. 2011, 74, 124513. [CrossRef]

3. Gofryk, K.; Pan, M.; Cantoni, C.; Saparov, B.; Mitchell, J.E.; Sefat, A.S. Local inhomogeneity and filamentary superconductivity in Pr-doped $\mathrm{CaFe}_{2} \mathrm{As}_{2}$. Phys. Rev. Lett. 2014, 112, 047005. [CrossRef] [PubMed]

4. Zeljkovic, I.; Huang, D.; Song, C.-L.; Lv, B.; Chu, C.-W.; Hoffman, J.E. Nanoscale surface element identification and dopant homogeneity in the high-T c superconductor $\operatorname{Pr}_{x} \mathrm{Ca}_{1-x} \mathrm{Fe}_{2} \mathrm{As}_{2}$. Phys. Rev. B 2013, 87, 201108. [CrossRef] 
5. Xiao, H.; Hu, T.; He, S.K.; Shen, B.; Zhang, W.J.; Xu, B.; He, K.F.; Han, J.; Singh, Y.P.; Wen, H.H.; et al. Filamentary superconductivity across the phase diagram of $\mathrm{Ba}(\mathrm{Fe}, \mathrm{Co})(2)$ As-2. Phys. Rev. B 2012, 86, 064521. [CrossRef]

6. Moseley, D.; Yates, K.A.; Branford, W.R.; Sefat, A.S.; Mandrus, D.; Stuard, S.J.; Salem-Sugui, S.; Ghivelder, L.; Cohen, L.F. Signatures of filamentary superconductivity in antiferromagnetic $\mathrm{BaFe}_{2} \mathrm{As}_{2}$ single crystals. Epl-Europhys Lett. $2015,111,37005$. [CrossRef]

7. Sefat, A.S. Bulk synthesis of iron-based superconductors. Curr. Opin. Solid St M 2013, 17, 59-64. [CrossRef]

8. Saha, S.R.; Butch, N.P.; Drye, T.; Magill, J.; Ziemak, S.; Kirshenbaum, K.; Zavalij, P.Y.; Lynn, J.W.; Paglione, J. Structural collapse and superconductivity in rare-earth-doped $\mathrm{CaFe}_{2} \mathrm{As}_{2}$. Phys. Rev. B 2012, 85, 024525. [CrossRef]

9. Qi, Y.; Gao, Z.; Wang, L.; Wang, D.; Zhang, X.; Yao, C.; Wang, C.; Wang, C.; Ma, Y. Transport properties and anisotropy in rare-earth doped $\mathrm{CaFe}_{2} \mathrm{As}_{2}$ single crystals with Tc above $40 \mathrm{~K}$. Supercond. Sci. Technol. 2012, 25, 045007. [CrossRef]

10. Zhou, W.; Shi, Z. Review on origin of the unusual high-Tc superconductivity in $\mathrm{Ca}_{1-x} \mathrm{RExFe}_{2} \mathrm{As}_{2}(\mathrm{RE}=\mathrm{La}, \mathrm{Ce}, \mathrm{Pr}, \mathrm{Nd})$. Novel Supercond. Mater. 2015, 1. [CrossRef]

11. Zou, Q.; Wu, Z.M.; Fu, M.M.; Zhang, C.M.; Rajput, S.; Wu, Y.P.; Li, L.; Parker, D.S.; Kang, J.; Sefat, A.S.; et al. Effect of Surface Morphology and Magnetic Impurities on the Electronic Structure in Cobalt-Doped $\mathrm{BaFe}_{2} \mathrm{As}_{2}$ Superconductors. Nano Lett. 2017, 17, 1642-1647. [CrossRef] [PubMed]

12. Sefat, A.S.; Nguyen, G.D.; Parker, D.S.; Fu, M.M.M.; Zou, Q.; Li, A.P.; Cao, H.B.B.; Sanjeewa, L.D.; Li, L.; Gai, Z. Local superconductivity in vanadium iron arsenide. Phys. Rev. B 2019, 100, 104525. [CrossRef]

13. Saha, S.; Butch, N.; Kirshenbaum, K.; Paglione, J.; Zavalij, P. Superconducting and ferromagnetic phases induced by lattice distortions in stoichiometric $\mathrm{SrFe}_{2} \mathrm{As}_{2}$ single crystals. Phys. Rev. Lett. 2009, 103, 037005. [CrossRef] [PubMed]

14. Engelmann, J.; Grinenko, V.; Chekhonin, P.; Skrotzki, W.; Efremov, D.; Oswald, S.; Iida, K.; Hühne, R.; Hänisch, J.; Hoffmann, M. Strain induced superconductivity in the parent compound $\mathrm{BaFe}_{2} \mathrm{As}_{2}$. Nat. Commun. 2013, 4, 2877. [CrossRef] [PubMed]

15. Xiao, H.; Hu, T.; Dioguardi, A.; Shockley, A.; Crocker, J.; Nisson, D.; Viskadourakis, Z.; Tee, X.; Radulov, I.; Almasan, C. Evidence for filamentary superconductivity nucleated at antiphase domain walls in antiferromagnetic $\mathrm{CaFe}_{2} \mathrm{As}_{2}$. Phys. Rev. B 2012, 85, 024530. [CrossRef]

16. Ozaki, T.; Wu, L.; Zhang, C.; Jaroszynski, J.; Si, W.; Zhou, J.; Zhu, Y.; Li, Q. A route for a strong increase of critical current in nanostrained iron-based superconductors. Nat. Commun. 2016, 7, 13036. [CrossRef] [PubMed]

17. Mizuguchi, Y.; Hara, Y.; Deguchi, K.; Tsuda, S.; Yamaguchi, T.; Takeda, K.; Kotegawa, H.; Tou, H.; Takano, Y. Anion height dependence of Tc for the Fe-based superconductor. Supercond. Sci. Technol. 2010, 23, 054013. [CrossRef]

18. Deng, L.; Lv, B.; Zhao, K.; Wei, F.; Xue, Y.; Wu, Z.; Chu, C. Evidence for defect-induced superconductivity up to $49 \mathrm{~K}$ in $\left(\mathrm{Ca}_{1-\mathrm{x}} \mathrm{R}_{\mathrm{x}}\right) \mathrm{Fe}_{2} \mathrm{As}_{2}$. Phys. Rev. B 2016, 93, 054513. [CrossRef] 